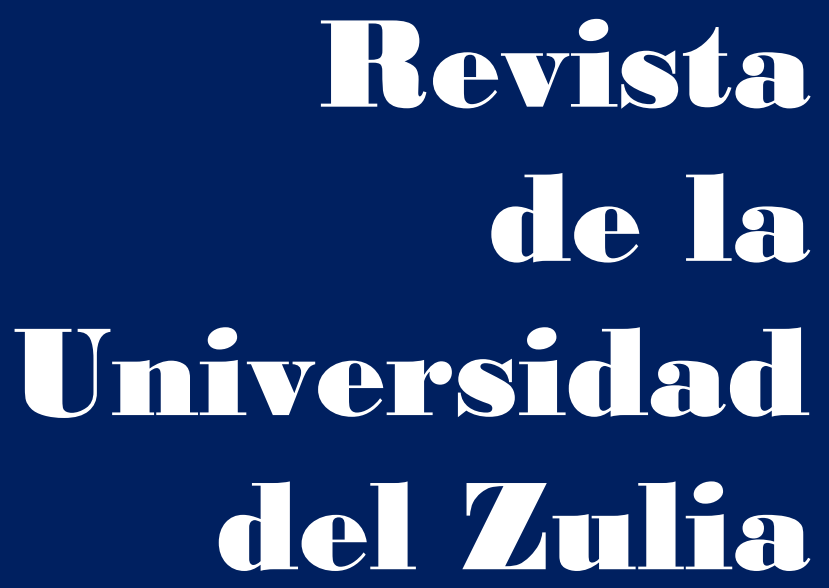

Fundada en 1947

por el Dr. Jesús Enrique Lossada

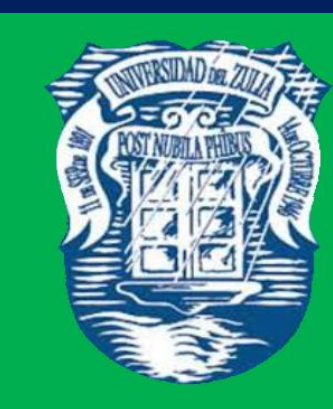

Ciencias del

Agrad,

Ingemiería

y Tecinología

\section{Aกัต 13 No $\mathbf{3 6}$} Enero - Abril 2022

Tercera Épaca

Maracailbo-Venezuela 
REVISTA DE LA UNIVERSIDAD DEL ZULIA. $3^{a}$ época. Año $13 \mathrm{~N}^{\circ}$ 36, 2022

J. E. Abanto Cubas et al. /// Calidad de agua de lluvia en prototipos de captación... 189-199

DOI: http://dx.doi.org/10.46925//rdluz.36.13

\title{
Calidad de agua de lluvia en prototipos de captación en las comunidades nativas de Tunants y Yahuahua, Amazonas-Perú
}

\author{
Jheny Elizabeth Abanto Cubas* \\ Eli Morales Rojas ** \\ Edwin Adolfo Díaz Ortiz ${ }^{* * *}$
}

\begin{abstract}
RESUMEN
El objetivo de la investigación consistió en determinar la calidad fisicoquímica y microbiológica del agua de lluvia de los cuatro prototipos de captación, instalados en las comunidades nativas de Tunants y Yahuahua, en el departamento de Amazonas, Perú. Se establecieron puntos de muestreo, dos por cada prototipo (entrada y salida); las evaluaciones se realizaron en los meses de febrero, septiembre y octubre del 2020. Se evaluaron parámetros de pH, Turbidez, Coliformes Totales (CT), Coliformes Termotolerantes (CTr), Aluminio (Al) y Zinc (Zn). Los resultados arrojaron valores de $\mathrm{pH}$ 7.16; turbidez 1.58 UNT. Estos fueron inferiores a los establecidos por el D.S. № 031- 2010- SA; en cuanto a los análisis microbiológicos de CT y CF, se obtuvo valores de 212.5 y 387.5 UFC/100 ml que superan los límites máximos permisibles. Se concluye que el agua de lluvia examinada requiere para su consumo el hervido o desinfección continua con hipoclorito de sodio u otro desinfectante, a fin de asegurar la salubridad de la población y evitar problemas gastrointestinales.
\end{abstract}

PALABRAS CLAVE: agua potable; tratamiento del agua; calidad del agua; Perú.

* Facultad de Ingeniería Civil y Ambiental, Universidad Nacional Toribio Rodríguez de Mendoza de Amazonas, Perú. ORCID: https://orcid.org/0000-0001-6718-421X. E-mail: 7143529942@untrm.edu.pe

** Investigador del Instituto de Investigación para el Desarrollo Sustentable de Ceja de Selva (INDES-CES), Universidad Nacional Toribio Rodríguez de Mendoza de Amazonas, Perú. ORCID: https://orcid.org/0000-0002-8623-3192. E-mail: eli.morales@untrm.edu.pe

*** Investigador de la Facultad de Ingeniería Civil y Ambiental, Universidad Nacional Toribio Rodríguez de Mendoza de Amazonas, Perú. ORCID: https:/orcid.org/0000-00017213-9552. E-mail: edwin.diaz@untrm.edu.pe 
REVISTA DE LA UNIVERSIDAD DEL ZULIA. $3^{a}$ época. Año $13 \mathrm{~N}^{\circ}$ 36, 2022

J. E. Abanto Cubas et al. /// Calidad de agua de lluvia en prototipos de captación... 189-199

DOI: http://dx.doi.org/10.46925//rdluz.36.13

\section{Rainwater quality in catchment prototypes in the native communities of Tunants and Yahuahua, Amazonas-Peru}

ABSTRACT

The objective of the research was to determine the physicochemical and microbiological quality of the rainwater of the four catchment prototypes, installed in the native communities of Tunants and Yahuahua, in the department of Amazonas, Peru. Sampling points were established, two for each prototype (entry and exit); The evaluations were carried out in the months of February, September and October 2020. Parameters of pH, Turbidity, Total Coliforms (TC), Thermotolerant Coliforms (TrC), Aluminum (Al) and Zinc $(\mathrm{Zn})$ were evaluated. The results showed values of $\mathrm{pH} 7.16$; turbidity $1.58 \mathrm{NTU}$. These were lower than those established by the D.S. No. 031-2010-SA; Regarding the microbiological analyzes of CT and CF, values of 212.5 and $387.5 \mathrm{CFU} / 100 \mathrm{ml}$ were obtained that exceed the maximum permissible limits. It is concluded that the rainwater examined requires continuous boiling or disinfection with sodium hypochlorite or another disinfectant for consumption, in order to ensure the health of the population and avoid gastrointestinal problems.

KEY WORDS: Drinking water; Water treatment; Water quality; Peru.

\section{Introducción}

La calidad del agua, la salud y el crecimiento económico son fundamentales para el desarrollo del ser humano (Villena,2018). Sin embargo, el agua para consumo humano está cada vez más amenazada por los contaminantes que genera la humanidad y la disminución del recurso hídrico debido al calentamiento global (Ripple et al.,2017). El crecimiento poblacional y las condiciones físicas y químicas de los suelos, producto de las escorrentías superficiales y subterráneas deterioran la calidad del agua (Álvarez et al., 2008). Es por ello que los contaminantes de agua incluyen microorganismos bacterianos, virales, fúngicos y parasitarios; adicionales a sustancias químicas simples o complejas como metales pesados, sustancias radiactivas, insecticidas, fertilizante, entre otras que afectan a la salud (Gómez, 2018).

Según Morell y Hernández (2000), la concentración de contaminantes en el agua limita la viabilidad del líquido y aumenta su grado de toxicidad para el ser humano. De acuerdo con el informe Año Internacional del Saneamiento, publicado en el año 2008, la 
REVISTA DE LA UNIVERSIDAD DEL ZULIA. $3^{a}$ época. Año $13 \mathrm{~N}^{\circ}$ 36, 2022

J. E. Abanto Cubas et al. /// Calidad de agua de lluvia en prototipos de captación... 189-199

DOI: http://dx.doi.org/10.46925//rdluz.36.13

quinta parte de la población a nivel mundial no tiene acceso a agua libre de contaminantes (ONU-Agua, 2008). La calidad del agua se determina comparando las características fisicoquímicas y microbiológicas del agua de acuerdo a protocolos o estándares de calidad (Quiñones, 2016).

Entre los parámetros fisicoquímicos se encuentra el potencial de hidrogeno $\mathrm{pH}$, en química es una escala numérica utilizada para especificar la acidez o alcalinidad de una solución (Vázquez y Rojas, 2016); la turbidez que mide la cantidad de partículas suspendidas en el agua, metales pesados como aluminio y zinc; se considera que el incremento en la concentración de estos metales en las fuentes hídricas procede de las diversas actividades antropogénicas, causando efectos nocivos en los sistemas ecológicos y salud (Pabón et al., 2020).

Dentro de los parámetros microbiológicos tenemos el grupo coliformes que están presentes en el ambiente, plantas, suelos y el tracto digestivo de los animales y humanos ("Bacterias Coliformes en el Agua", 2021). Estos se clasifican en Coliformes Totales y Coliformes Termotolerantes; la mayoría de estas bacterias son inofensivas para el ser humano; los síntomas más comunes son malestar gastrointestinal, síntomas de fiebre, calambres abdominales y diarrea ("Bacterias Coliformes", 2020). Es por ello que es importante y necesario realizar estudios de calidad de agua, con fines de salvaguardar la salud de la población.

Sobre la base de lo mencionado, el objetivo de este estudio fue evaluar la calidad fisicoquímica, microbiológica del agua de lluvia colectada de prototipos de captación, en dos comunidades nativas Tunants y Yahuahua pertenecientes a la región Amazonas- Perú.

\section{Materiales y métodos}

El estudio se realizó en cuatro viviendas de las comunidades de Tunants y Yahuahua de la selva alta del Perú, departamento Amazonas, provincia Condorcanqui, distrito de Nieva, con localización en las coordenadas geográficas UTM-WGS84 Este 180917.44 y Norte 9492 371.00; con una altitud de 230 m.s.n.m (PVPP, 2009); tiene una temperatura media anual entre 22 y $26^{\circ} \mathrm{C}$, con precipitaciones anuales entre $2600 \mathrm{~mm}$ a $4000 \mathrm{~mm}$ (MIDAGRI, s.f.) (Figura 1). 


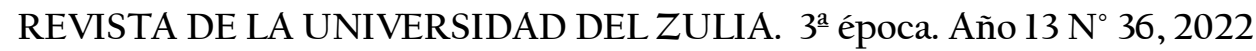

J. E. Abanto Cubas et al. /// Calidad de agua de lluvia en prototipos de captación... 189-199 DOI: http://dx.doi.org/10.46925//rdluz.36.13

Para todos los análisis fisicoquímicos, microbiológicos y metales pesados, se siguió la metodología establecida en el American Public Health Association (APHA, AWWA, \& WPFC, 2005). Para el análisis del pH se utilizó un multiparámetro según el método 4500-H; la turbidez se analizó mediante un Turbidímetro, según el método USEPA 180.1. Los análisis microbiológicos, grupo coliformes, se determinaron en dos fases: fase presuntiva, "el medio de cultivo que se utilizó es el caldo de Lauril Sulfato, el cual permite que los microorganismos presentes en el agua se desarrollen utilizando la lactosa como fuente de alimento" (Camacho et al., 2009); y la fase confirmativa, que se realizó a partir de los tubos positivos que se comprueban en la fase presuntiva, para ello el medio de cultivo que se utiliza es Brilla para confirmación de Coliformes Totales y Ec para confirmación de Coliformes Termotolerantes (Figura 2).

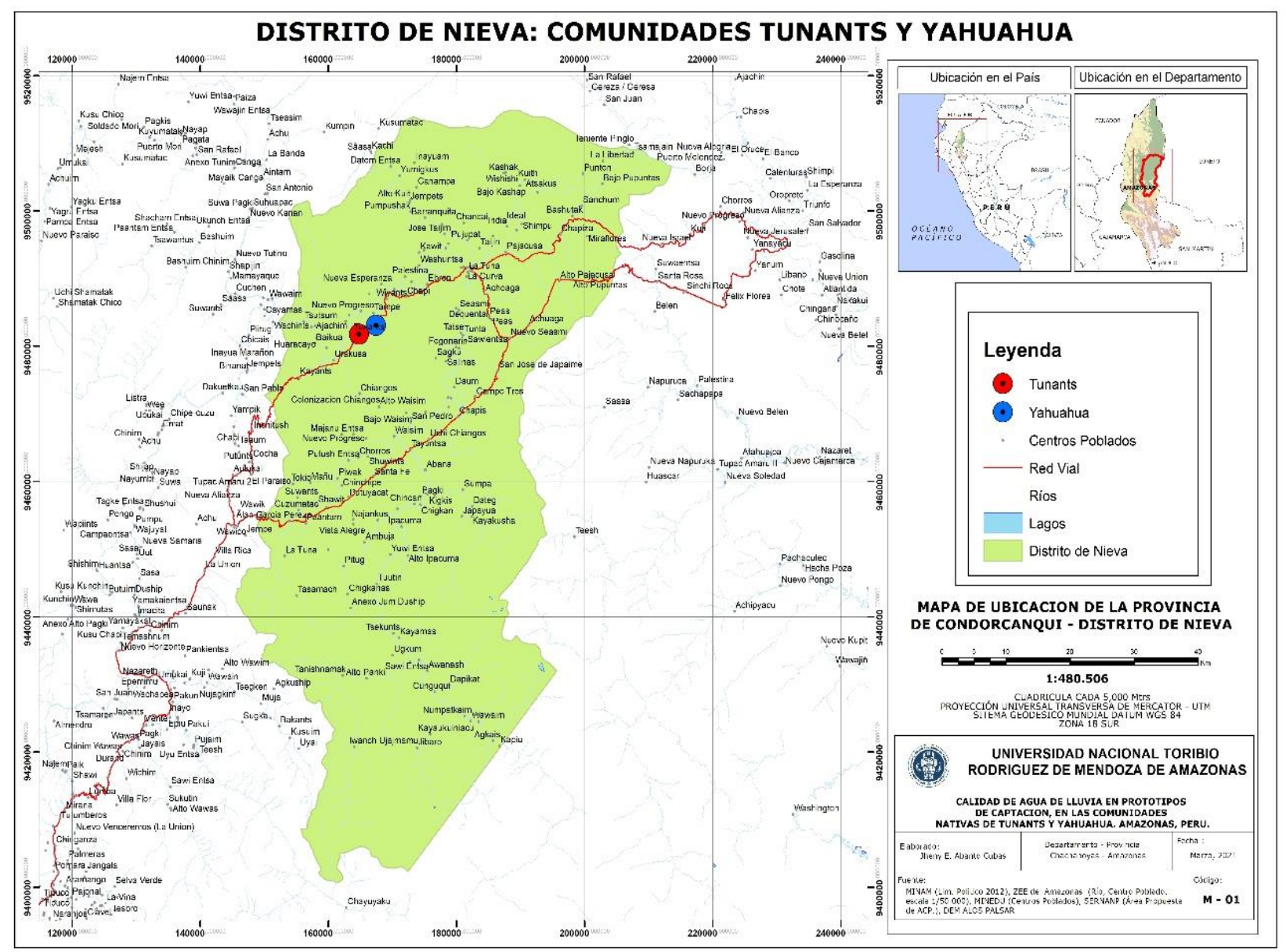

Figura l. Mapa de ubicación de la provincia de Condorcanqui, distrito Nieva. 
REVISTA DE LA UNIVERSIDAD DEL ZULIA. $3^{a}$ época. Año $13 \mathrm{~N}^{\circ}$ 36, 2022

J. E. Abanto Cubas et al. /// Calidad de agua de lluvia en prototipos de captación... 189-199 DOI: http://dx.doi.org/10.46925//rdluz.36.13

Los parámetros de metales pesados (aluminio, zinc), se determinaron mediante un proceso de filtración y se agregó 0,3 ml de ácido nítrico para su conservación. Las lecturas se efectuaron con un Espectrofotómetro de Barrido marca ERMO SCIENTI. Los análisis se realizaron en el Laboratorio de Aguas y Suelos del Instituto de Investigación para el Desarrollo Sustentable de Ceja de Selva (INDES-CES) de la Universidad Nacional Toribio Rodríguez de Mendoza de Amazonas. Todos los parámetros fueron comparados de acuerdo al Artículo 63, Parámetros de control obligatorio (PCO), del Decreto Supremo № 031-2010S.A.

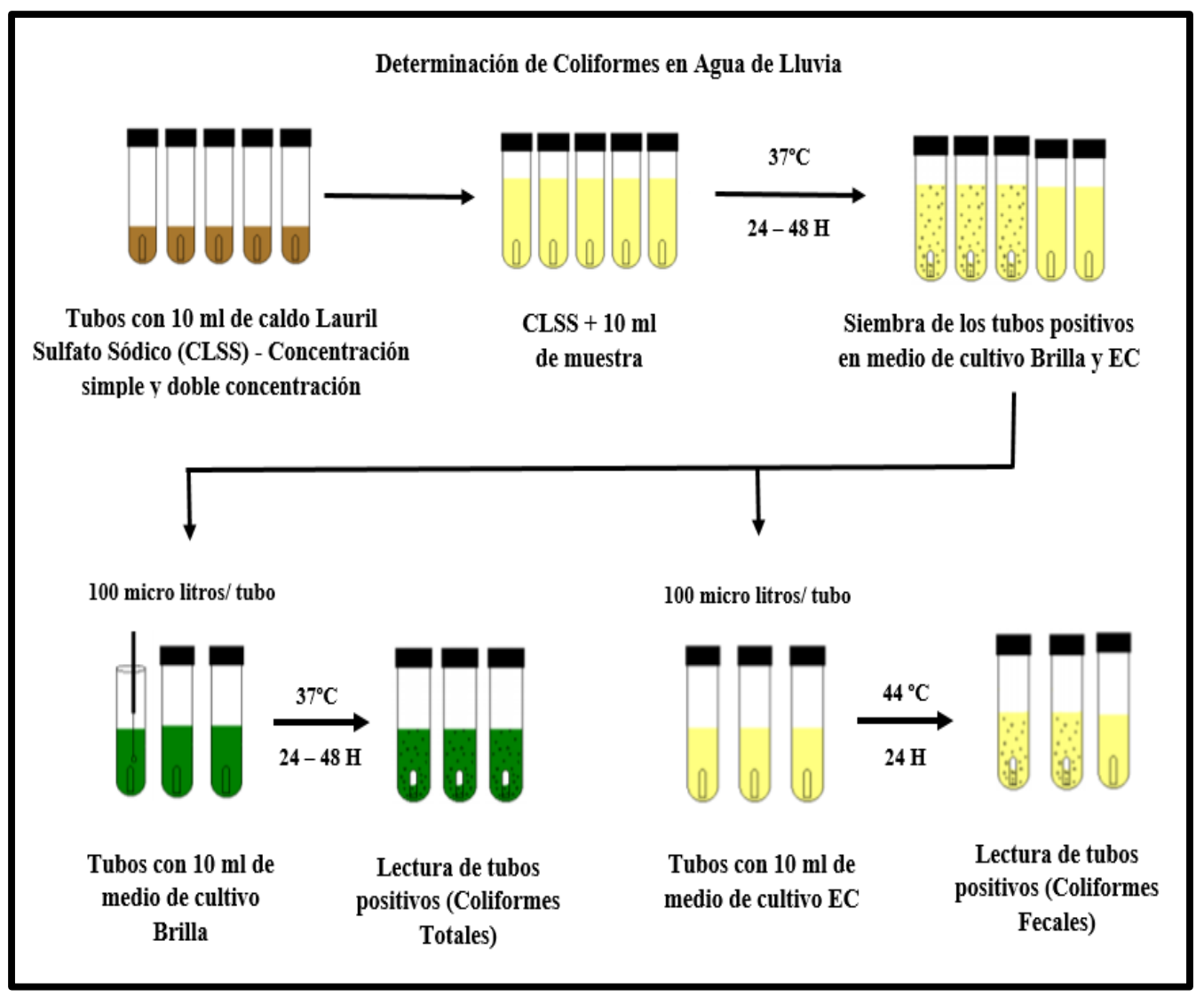

Figura 2: Proceso para determinación de coliformes.

\subsection{Análisis de datos}

Mediante el test de normalidad de Shapiro-Wilk, se contrastó la normalidad de cada una de las variables en estudio; y mediante $\mathrm{T}$ de Student, se determinaron las diferencias significativas de los parámetros evaluados entre la entrada y salida de agua de lluvia, haciendo uso del software Minitab 17. 
REVISTA DE LA UNIVERSIDAD DEL ZULIA. $3^{a}$ época. Año $13 \mathrm{~N}^{\circ}$ 36, 2022

J. E. Abanto Cubas et al. /// Calidad de agua de lluvia en prototipos de captación... 189-199

DOI: http://dx.doi.org/10.46925//rdluz.36.13

\section{Resultados}

Los resultados indican que el $\mathrm{pH}$ a la entrada del prototipo obtuvo una mediana de 7,334 a diferencia del valor de salida que obtuvo una mediana de 7,008, valores que se encuentran dentro de los Límites Máximos Permisibles (LMP); la turbidez arrojó una media de 1,616 UNT en entrada y 1.558 UNT en salida. En cuanto a los análisis microbiológicos, los Coliformes Totales entrada obtuvo una mediana de $258 \mathrm{UFC} / 100 \mathrm{ml}$, y de salida una mediana de 167 UFC/100 ml; al igual que los Coliformes Termotolerantes, entrada obtuvo una mediana de 637 UFC/100 ml, y de salida una mediana de 138 UFC/100 ml; ambos grupos coliformes obtuvieron valores que superan los Límites Máximos Permisibles (LMP). En los análisis de metales pesados como aluminio entrada se obtuvo una mediana de $0,274 \mathrm{mg} \mathrm{Al} \mathrm{L}^{1}$ y de salida 0,1458 $\mathrm{mg} \mathrm{Al} \mathrm{L}^{-1}$; y zinc entrada se obtuvo una mediana de 2,705 $\mathrm{mg} \mathrm{Zn} \mathrm{L}^{-1}$ y de salida 2.369 mg Zn L $L^{-1}$ valores inferiores a los límites máximos permisibles. Los datos fueron equivalentes a p > 0,05; estos indican que existe una distribución normal y no existe diferencia significativa entre las variables estudiadas. (Tabla 1).

Tabla l. Resultados de los análisis fisicoquímicos y microbiológicos.

\begin{tabular}{|c|c|c|c|c|c|c|}
\hline Parámetros & Unidad & Media & Desv.Est. & Valor T & Valor $\mathrm{p}$ & LMP \\
\hline $\mathrm{pH}(\mathrm{E})$ & & 7.334 & 0.936 & \multirow{2}{*}{1.23} & \multirow{2}{*}{0.246} & \multirow{2}{*}{$6.5-8.5$} \\
\hline $\mathrm{pH}(\mathrm{S})$ & & 7.008 & 0.197 & & & \\
\hline Turb (E) & \multirow[b]{2}{*}{ UNT } & 1.616 & 0.591 & \multirow{2}{*}{0.32} & \multirow{2}{*}{0.755} & \multirow{2}{*}{$5(\mathrm{UNT})$} \\
\hline Turb (S) & & 1.558 & 0.429 & & & \\
\hline $\mathrm{CT}(\mathrm{E})$ & \multirow{2}{*}{$\begin{array}{c}\mathrm{NMP} / 100 \\
\mathrm{~mL}\end{array}$} & $258^{*}$ & 494 & \multirow{2}{*}{0.61} & \multirow{2}{*}{0.555} & \multirow{2}{*}{ «1.8 (NMP) } \\
\hline CT (S) & & $167^{*}$ & 462 & & & \\
\hline $\mathrm{CTr}(\mathrm{E})$ & \multirow{2}{*}{$\begin{array}{c}\mathrm{NMP} / 100 \\
\mathrm{~mL}\end{array}$} & $637^{*}$ & 728 & \multirow{2}{*}{2.55} & \multirow{2}{*}{0.027} & \multirow{2}{*}{$<1.8$ (NMP) } \\
\hline $\mathrm{CTr}(\mathrm{S})$ & & $138^{*}$ & 460 & & & \\
\hline Aluminio (E) & \multirow{2}{*}{$\mathrm{mg} \mathrm{Al} \mathrm{L-1}$} & 0.2748 & 0.3236 & \multirow[b]{2}{*}{1.27} & \multirow[b]{2}{*}{0.231} & \multirow[b]{2}{*}{$0.2(\mathrm{mg} \mathrm{Al} \mathrm{L}-1)$} \\
\hline Aluminio (S) & & 0.1685 & 0.1458 & & & \\
\hline Zinc (E) & \multirow{2}{*}{ mg Zn L-1 } & 2.705 & 1.944 & \multirow[b]{2}{*}{0.94} & \multirow[b]{2}{*}{0.366} & \multirow[b]{2}{*}{$3(m g Z n L-1)$} \\
\hline Zinc (S) & & 2.369 & 2.087 & & & \\
\hline
\end{tabular}

LMP: Límites Máximos Permisibles; * Valores que no cumplen con la normativa reportado en el Articulo 63. Parámetros de control obligatorio (PCO). Decreto Supremo № 031-2010-S.A. 
REVISTA DE LA UNIVERSIDAD DEL ZULIA. $3^{a}$ época. Año $13 \mathrm{~N}^{\circ}$ 36, 2022

J. E. Abanto Cubas et al. /// Calidad de agua de lluvia en prototipos de captación... 189-199

DOI: http://dx.doi.org/10.46925//rdluz.36.13

En la Figura l se observa el comportamiento de los parámetros en entrada y salida del prototipo de captación, siendo los Coliformes Termotolerantes y Coliformes Totales con mayor concentración en la entrada y concentración baja en la salida, debido a que se aplicó hipoclorito de sodio al 5 \%, con una concentración de l gota por litro, para su desinfección y consumo.

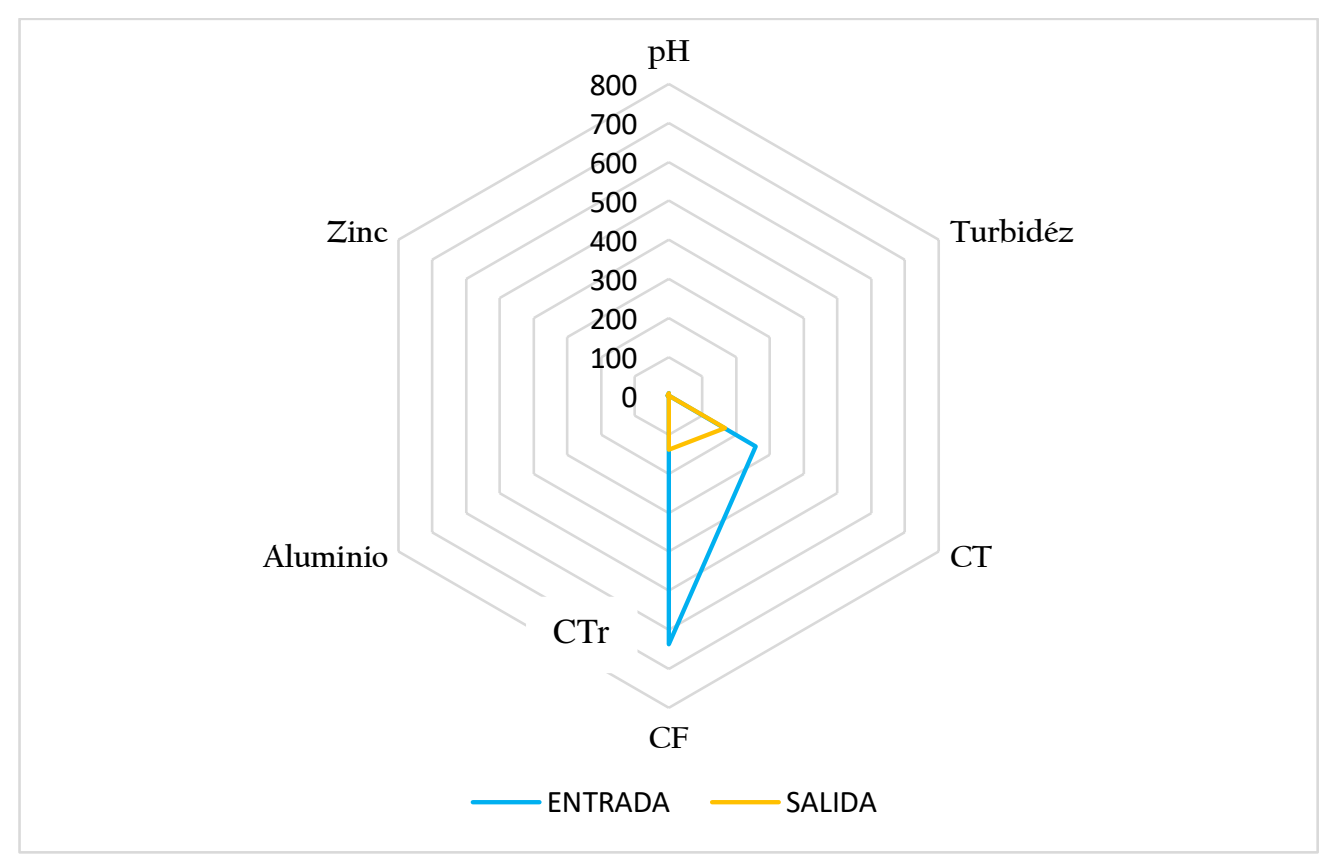

Figura 1. Comportamiento de los parámetros en entrada y salida del prototipo de captación; CTr=Coliformes Termotolerantes; $\mathrm{CT}=$ Coliformes Totales

\section{Discusión}

El $\mathrm{pH}$ en los prototipos evaluados tuvo un valor promedio de media de 7,16 valor que se encuentra dentro del Límite Máximo Permitido según el D.S. № 031-2010-S. A, que coincide con las investigaciones de Sazakli et al. (2007), quienes evalúan la calidad de agua subterránea o mezclada con agua de lluvia y calidad de agua de lluvia en la isla de Cefalonia, Grecia; donde los valores de pH oscilaron entre 7,63 y 8,80 con una media de 8, 35, siendo el valor del pH mayor en el agua de lluvia, encontrándose dentro de los límites establecidos por la directiva 98/93/UE.

En relación a la turbidez, se obtuvo un valor promedio de media de 1,58 UNT, encontrándose dentro del límite máximo permisible; lo que concuerda con las investigaciones de Minju et al. (2017), quienes evaluaron la calidad del agua de lluvia sin tratar y agua de lluvia 
REVISTA DE LA UNIVERSIDAD DEL ZULIA. $3^{a}$ época. Año $13 \mathrm{~N}^{\circ}$ 36, 2022

J. E. Abanto Cubas et al. /// Calidad de agua de lluvia en prototipos de captación... 189-199

DOI: http://dx.doi.org/10.46925//rdluz.36.13

con tratamiento para consumo en Vietnam, donde los valores de turbidez oscilaron entre 1 UNT y 0,3 UNT, siendo el valor de la turbidez bajo en el agua de lluvia tratada, encontrándose dentro de los estándares de agua potable de Vietnam.

En el caso de la calidad microbiana del agua de lluvia, los Coliformes Totales y Coliformes Termorolerantes obtuvieron valores promedio de media de 212,5 UFC/100 ml y 387,5 UFC/ $100 \mathrm{ml}$ superando ambos valores los límites máximos permisibles. Estos resultados guardan relación con lo que sostienen Minju et al. (2017), quienes obtuvieron un valor de 270 UFC/100 ml en coliformes y 8 UFC/100 ml de Escherichia Coli en todas las muestras de agua de lluvia sin tratar, "siendo los principales causantes de enfermedades gastrointestinales que influyen en la desnutrición infantil" (Medina et al.,2019).

En lo que respecta a los metales pesados, el aluminio obtuvo un valor promedio de media de 0,22 mg Al L-1, lo que es preocupante ya que su ingesta podría causar anemia y desnutrición crónica infantil (Cárdenas y Merma, 2018). Finalmente, el valor promedio de media de zinc fue de 2,54 mg Zn L-1, valor que se encuentra dentro de los límites máximos permisibles, algo que no coincide con las investigaciones de Mao et al. (2020), quienes evaluaron el efecto de los materiales del techo y los patrones climáticos en la calidad del agua de lluvia en Shanghái, obteniendo valores de Zn en el techo de material galvanizado, valores que oscilan entre 397 mg Zn L-1 y 1970 mg Zn L-1. Sin embargo, comparado con otros tipos de material para techos, el techo de metal galvanizado produce fácilmente la contaminación con metales pesados durante el uso a largo plazo (Zhang et al.,2014), ya que consta de una capa galvanizada en la superficie y una capa de hierro en el interior.

\section{Conclusiones}

Los parámetros fisicoquímicos evaluados como: $\mathrm{pH}$, turbidez, aluminio y zinc obtuvieron valores 7,16; 1,58 (UTN); 0,22 mg Al L-1 y 2,54 mg Zn L-1, valores que están dentro de los Límites Máximos Permisibles, asegurando la calidad del agua de lluvia.

Los valores de Coliformes Totales y Coliformes Termotolerantes presentan valores de 212,5 UFC /100 ml y 387,5 UFC /100 ml, valores que no cumplen con los Límites Máximos Permisibles, como lo establece la normativa en el Articulo 63, Parámetros de control obligatorio (PCO), Decreto Supremo № 031-2010-S.A., siendo necesario para su consumo el 
REVISTA DE LA UNIVERSIDAD DEL ZULIA. $3^{a}$ época. Año $13 \mathrm{~N}^{\circ}$ 36, 2022

J. E. Abanto Cubas et al. /// Calidad de agua de lluvia en prototipos de captación... 189-199

DOI: http://dx.doi.org/10.46925//rdluz.36.13

hervido o desinfección continua con hipoclorito de sodio u otro desinfectante, que aseguren la salubridad de la población y poder así evitar problemas gastrointestinales.

\section{Agradecimientos}

Al Proyecto de Investigación "Prototipos de sistema de potabilización del agua de lluvia en comunidades nativas del departamento de Amazonas. Perú 2019-2020" (PROLLUVIA), al Instituto de Investigación para el Desarrollo Sustentable de Ceja de Selva (INDES-CES) de la Universidad Nacional Toribio Rodríguez de Mendoza de Amazonas, por el apoyo logístico para realizar la presente investigación, así como al FONDECYT por el financiamiento del proyecto de investigación mediante contrato de subvención № 185-2018FONDECYT-BM-IADT-SE.

\section{Referencias}

Álvarez J., Panta j., Ayala C. y Acosta E. (2008). Calidad integral del agua superficial en la cuenca hidrológica del río Amajac. Información tecnológica. 19(6). http://dx.doi.org/10.4067/S071807642008000600004

American Public Health Association (APHA), American Water Work Association (AWWA) y Water Pollution Control Federation (WPFC). (2005). Standard Methods for the Examination of Water and Wastewater. Washington, Estados Unidos. Copyright.

Bacterias coliformes. (19 de octubre, 2020). Obtenido de https://extension.psu.edu/bacteriascoliformes

Bacterias coliformes en el agua. (9 de febrero, 2021). Obtenido de https://www.carbotecnia.info/aprendizaje/desinfeccion/bacterias-coliformes-en-el-aguapotable/

Bautista A., Tovar J., Mancilla O., Magdaleno H., Ramírez C., Arteaga R. y Vázquez M. (2013). Calidad microbiólogica del agua obtenida por condensación de la atmósfera en Tlaxcala, Hidalgo y ciudad de México. Revista Internacional de Contaminación Ambiental. 29(2). 167-175.

Camacho, A., M. Giles, A. Ortegón, M. Palao, B. Serrano y O. Velázquez (2009). Técnicas para el Análisis Microbiológico de Alimentos, México: Facultad de Química, UNAM.

Cárdenas C. y Merma V. (14 de diciembre de 2018). Espinar: Detectan presencial de metales tóxicos en agua de consumo humano. Ojo Público. https://ojo-publico.com/1019/espinardetectan-presencia-de-metales-toxicos-en-agua-de-consumo-humano

DIGESA (2015). Protocolo de procedimientos para la toma de muestras, preservación, conservación, transporte, almacenamiento y recepción de agua para consumo humano. Recuperado 
REVISTA DE LA UNIVERSIDAD DEL ZULIA. $3^{a}$ época. Año $13 \mathrm{~N}^{\circ}$ 36, 2022

J. E. Abanto Cubas et al. /// Calidad de agua de lluvia en prototipos de captación... 189-199

DOI: http://dx.doi.org/10.46925//rdluz.36.13

http://www.digesa.minsa.gob.pe/NormasLegales/Normas/RD_160_2015_DIGESA.pdf.

Gómez Duarte Oscar (2018). Contaminación de agua en países de bajos y medianos recursos es un problema de salud pública global. Revista de la Facultad de Medicina. 66(1). http://dx.doi.org/10.15446/revfacmed.v66nl.70775

Mao J., Xia B., Zhou Y., Bi F., Zhang X., Zhang W. y Xia S. (2020). Effect of roofing materials and weather patterns on the quality of collected rainwater in Shanghai, China. Revista de producción más limpia. 279. https://doi.org/10.1016/j.jclepro.2020.123419

Medina A., Mayca J., Velásquez J. y Llanos L. (2019). Conocimiento, percepciones y practices sobre el consumo de micronutrients en niños Awajún y Wampis (Condorcanqui, AmazonasPerú). Revista Acta Médica Peruana.36(3). 185-94

Ministerio de Desarrollo Agrario y Riego (s.f). MIDAGRI. https://www.minagri.gob.pe/portal/53-sector-agrario/el-clima

Minju L., Mikyeong K., Yonghwan K. y Mooyoung H. (2017). Consideration of rainwater quality parameters for drinking purposes: A case study in rural Vietnam. Revista de Gestión Ambiental. 200(15). 400-406. https://doi.org/10.1016/j.jenvman.2017.05.072

Morell I. y Hernández F. (2000). El agua en Castellón: Un reto para el siglo XXI. Castellón de la Plana: Editorial de la Universidad Jaume. España.

Organización de las Naciones Unidas (ONU) - Agua (2008), Hacia la solución de una crisis mundial: Año Internacional del Saneamiento. http:// esa.un.org/iys/docs/flagship_ES.pdf

Pabón E., Benítez R., Sarria R. y Gallo J. (2020). Contaminación del agua por metales pesados, métodos de análisis y tecnologías de remoción. Revista Entre ciencia e Ingeniería. 14(27). 9-18.

Plan Vial Provincial $\quad$ Participativo $\quad$ (2009). $\quad$ PVPP. http://www.proviasdes.gob.pe/planes/amazonas/pvpp/PVPP_Condorcanqui.pdf

Quiñones Huatangari L. (2016). Características Fisicoquímicas y microbiológicas del agua superficial del bosque de Chinchiquilla, Nueva Libertad, Chirinos, Cajamarca. Revista Científica Pakamuros. 4(7). 10.37787/pakamuros-unj.v4il.39

Ripple William, Wolf Christopher, Newsome Thomas, Galetti Mauro, Alamgir Mohammed, Crist Eileen, Mahmound I y Laurance William (2017). Advertencia de los científicos mundiales a la humanidad: un segundo aviso. Revista BioScience, 67(12), 1026-1028. https://doi.org/10.1093/biosci/bixl25

Sazakli E., Alexopoulos A. y Leotsinidis M. (2007). Rainwater harvesting, quality assessment and utilization in Kefalonia Island, Greece. Revista ELSEVIER. 4l(9). 2039-2047. https://doi.org/10.1016/j.watres.2007.01.037

Vázquez E. y Rojas T. (2016). pH: Teoría y 232 problemas. Universidad Autónoma Metropolitana.

Villena Chávez Jorge A. (2018). Calidad del agua y desarrollo sostenible. Revista peruana de medicina 
REVISTA DE LA UNIVERSIDAD DEL ZULIA. $3^{a}$ época. Año $13 \mathrm{~N}^{\circ}$ 36, 2022

J. E. Abanto Cubas et al. /// Calidad de agua de lluvia en prototipos de captación... 189-199

DOI: http://dx.doi.org/10.46925//rdluz.36.13

experimental y salud pública. 35(2). https://doi.org/10.17843/rpmesp.2018.352.3719

Zhang Q., Wang X., Hou P., Wan W., Li R., Ren Y. y Ouyang Z. (2014). Quality and seasonal variation of rainwater collected from concrete, asphalt, ceramic tile roofs and greens in Chongqing, China. Revista de Gestión Ambiental. 132. 178-187. https://doi.org/10.1016/j.jenvman.2013.11.009

\section{Anexos}

Anexo 1: Sistema de prototipo de captación y muestreo de agua de lluvia.
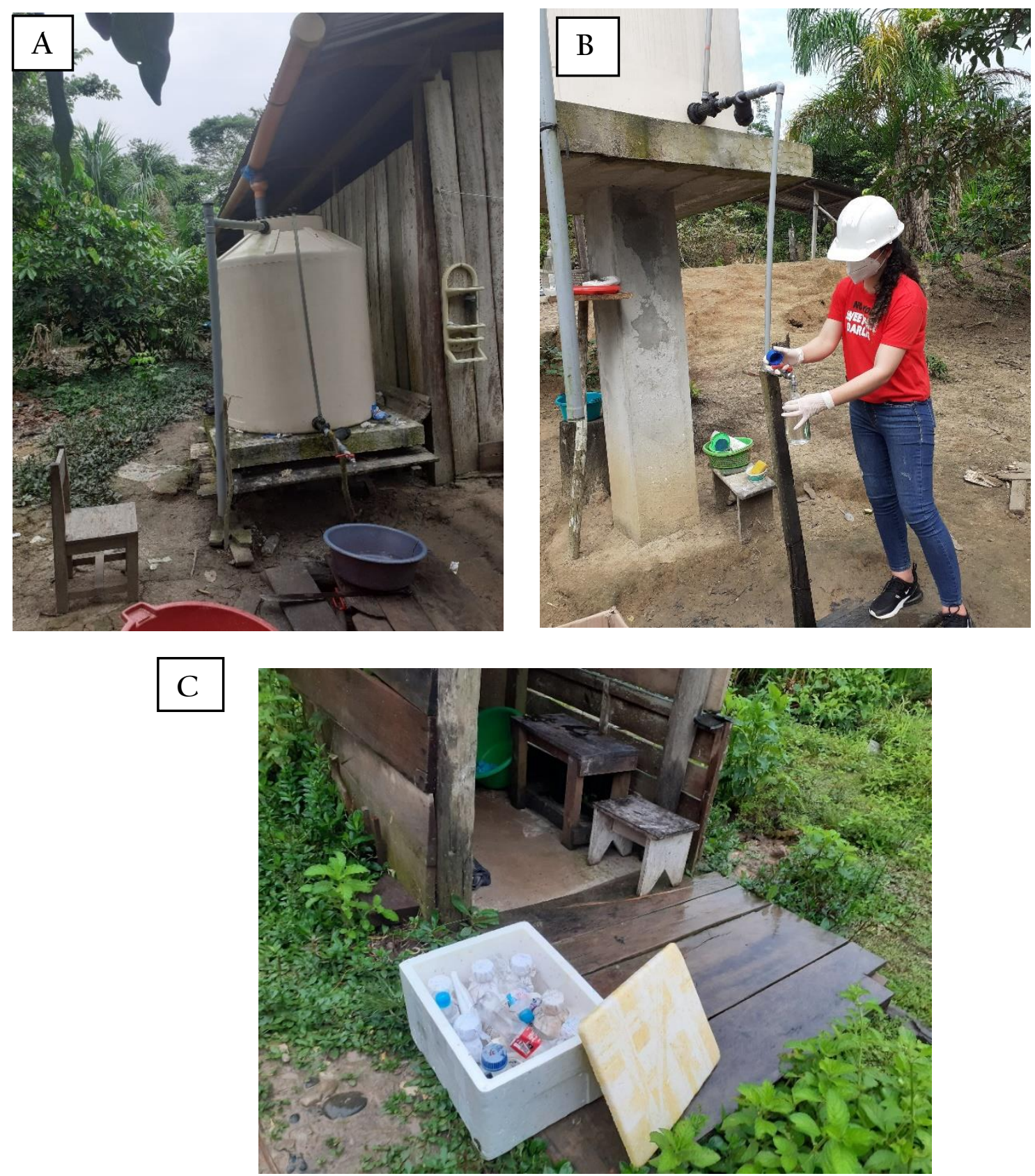

Figura A=Prototipo de captación y reutilización de agua de lluvia; Figura B=Recolección de muestras de agua; Figura $C=C a j a$ de tecnopor acondicionada con hielo seco para el traslado de muestras. 